\title{
Expanding the Teaching of Single Frequency Vibration Absorption to Broadband Attenuation using Subordinate Oscillator Arrays via Fettuccine Pasta
}

\author{
Sai Tej Paruchuri ${ }^{\mathrm{a}, 1, *}$, Vijaya V. N. Sriram Malladi ${ }^{\mathrm{b}, 1}$, Pablo A. Tarazaga ${ }^{\mathrm{a}}$, Andrew J. Kurdila ${ }^{\mathrm{a}}$ \\ ${ }^{a}$ Department of Mechanical Engineering, \\ Virginia Polytechnic Institute and State University, Blacksburg, Virginia, 24061 \\ ${ }^{b}$ Department of Mechanical Engineering- Engineering Science, \\ Michigan Technological University, Houghton, Michigan, 49931
}

\begin{abstract}
Dynamic vibration absorbers (DVAs) and tuned mass-dampers (TVAs) have wide-spread applications in the aerospace industry, the automotive sector, and in civil engineering structures. There are numerous designs of active and passive vibration attenuators or absorbers that isolate structural vibrations at or around the desired frequency. All these design approaches are fundamentally different ways to modify and tune the placement of the resonant frequencies of the host structure. The current work presents a novel method to passively attenuate vibration over a broad frequency bandwidth in the presence of uncertainty. An array of linear oscillators, also referred to as subordinate oscillator arrays (SOAs), are attached to a two-degrees-of-freedom structure to produce an attenuated broadband frequency response around a target frequency. SOAs can also be interpreted as an array of DVAs and in some categories, they can be considered as an approach to meta-structures.

Another objective of the current work is to develop a hands-on approach to extend classroom teaching of vibration-isolation using SOAs made out of fettuccine strands and modeling clay. The frequencies of the oscillators in the array are tuned by varying the length of each strand and the mass of the modeling clay attached to its tip. Uncertainty in dynamic properties of such oscillators often results in mistuned SOAs with non-uniform frequency response function. Therefore, designing and testing fettuccine-based SOAs allows students to handle cases when structural uncertainties arise in engineering systems. Additionally, some of the work in the field of meta-structures can be modeled and represented by SOAs and this will provide a straight forward way to teach students some of these contemporary concepts.
\end{abstract}

Keywords: Vibration Isolation, Subordinate Oscillator Array, Fettuccine Pasta, Modeling Clay, Structural Dynamics, Education, Dynamic Vibration Absorbers

\section{Introduction}

For more than a century, dynamic vibration absorbers (DVAs) have attenuated vibratory response near the natural frequency of engineering structures [1]. The fundamental idea behind DVAs is to improve the dynamics close to the resonance frequency of the host structure by securing a secondary dynamic system (a subordinate system) tuned to this frequency. Different applications have embraced and

\footnotetext{
${ }^{*}$ Corresponding author

Email address: saitejp@vt.edu (Sai Tej Paruchuri)

${ }^{1}$ These authors have contributed equally
} 
adopted subordinate systems with tuned spring-mass systems or tuned dynamic flywheels. Although these subordinate-oscillators have been widely employed in engineering structures, one limitation arises from its dependence on tuning to a specific frequency. As most linear DVAs are tuned to a frequency, the effectiveness of the dynamic absorber is limited to a narrow bandwidth. This can be impractical if (i) the natural frequency of the host structure changes, for instance, with operational loads, (ii) there is a mismatch between the resonant frequency of the structure and the tuned frequency of the DVA, and (iii) the host structure is under a broadband dynamic load. To address some of these drawbacks, researchers have developed tunable dynamic absorbers, nonlinear DVAs, active absorbers, and subordinate oscillator arrays (SOAs).

Subordinate oscillator arrays can be viewed as an array of linear DVAs that achieve vibration attenuation over a broad range of frequencies [2-6]. The isolated natural frequencies of the oscillators in the SOA are spaced around the natural frequencies of the host structure to achieve broadband attenuation. Various design approaches can accomplish this goal. The most straightforward method involves the placement of the isolated natural frequencies around the host structure's natural frequency in a random manner until the necessary attenuation is achieved over the required frequency range. A different approach would involve adding one oscillator at a time into the array corresponding to the highest observed peak in the desired frequency range and reevaluating the frequency response of the system each time. Naturally, this is a slower approach. However, it is highly efficient, and the SOAs designed using this approach have considerable fewer number of oscillators. Vignola et al. [6] proposed a method in which discrete distributions are assigned to the natural frequencies of the oscillators. As opposed to choosing individual parameters of oscillators, assigning distributions reduces the number of parameters needed to achieve the desired frequency distribution.

The performance of an SOA is highly dependent on the host and SOA uncertainty as well as the hosts structural properties. Vignola et al. [7] studied the effect of disorder on the SOA and showed that the SOAs performance deteriorated with an increasing error in the properties. The design approaches mentioned above do not take uncertainty in structural properties into consideration during the initial design phase. Arrays with piezoelectric oscillators, studied in [8-10], can adapt their effective structural properties in the presence of uncertainties. However, such an array of oscillators are complex to design and build when compared to ordinary SOAs.

In this paper, a design approach is adapted from the second method discussed above, that takes the uncertainties of the system into consideration and corrects the model to account for the disorder in the system. The effectiveness of this approach is tested using experiments on a two degree of freedom system, a physical model that mimics a two-story building. Fettuccine strands are used as cantilever beams for oscillators in the array. Since Young's modulus of fettuccine can vary from strand to strand, they make a suitable and inexpensive test subject for our approach.

Another objective of the present work is to bridge the gap between the research and the teaching communities via hands-on experimentation. Although many structural dynamics textbooks have discussed vibration absorbers $[11,12]$, there is a need for pedagogical clarity so that students can have a deeper understanding and appreciation of the underlying concepts. A hands-on approach that exemplifies the nuances of experimentally realizing a vibration absorber should be part of a traditional classroom learning process in vibrations. Therefore, the focus of the current effort is also to develop a strategy that assists in teaching principles behind vibration attenuation by extending classroom lectures through hands-on experience.

It should also be noted that the novel concepts of meta-structures can also be explained via this very simple example. For instance, Inman et al. develop a set of meta-structures in [13] that are simply a more sophisticated approach to the same concept. Although meta-structures can be elusive for entry level teaching and classes, an example such as this can relate the simple concepts of DVA and extend 


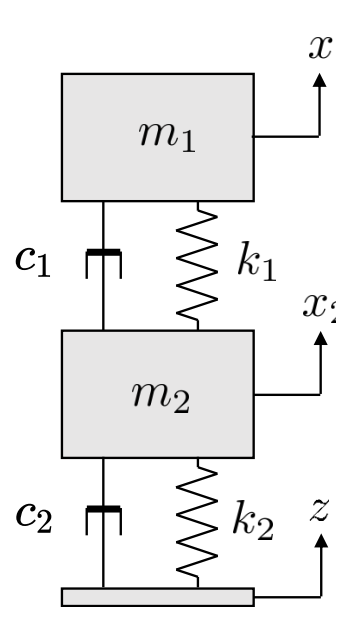

(a) 2-DOF Host Structure

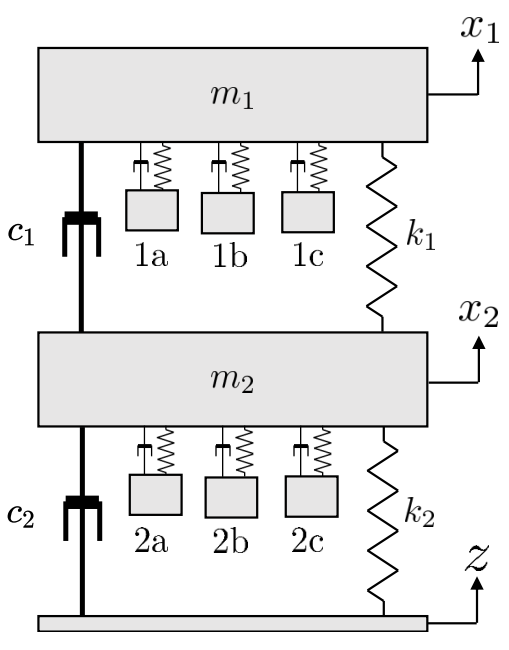

(b) Host Structure with SOAs

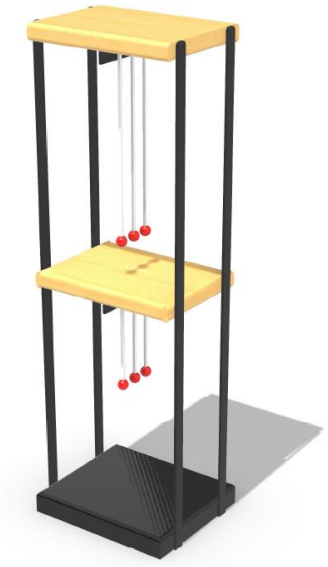

(c) $3 \mathrm{D}$ rendering of the structure

Figure 1: Host Structure

them to meta-structures.

As a part of this goal, SOAs are developed using non-conventional, yet mundane materials such as fettuccine and modeling clay. In designing tuned oscillators from such constituents, students have to apply theoretical formulations first to estimate approximate material properties and design oscillators to a particular frequency. The next step is to experimentally measure the oscillator's fundamental frequency, and observe the presence of uncertainty in the boundary conditions and material properties. This gives the students a chance to further tune the frequency by either reducing the length of the pasta or by adding tip-mass. Such an innovative and engaging hands-on approach compliments some of the recent efforts by the structural community to enhance the learning experience by using 3D printed technology [14] for teaching concepts of structural dynamics [15-18] through engaging experiments.

\section{Analytical model of a 2-DOF host structure}

This section presents the governing equations that model the 2-DOF structure shown in Figure 1. In later sections, SOAs are designed to target the natural frequencies of this representation of a twostory building. Figure 1 displays a 3D rendering of the host structure, along with a spring-mass-damper description of the structure. The 2-DOF system is modeled via a Lagrangian approach by determining the kinetic and potential energy of the host structure. Typically for a structure as in Figure 1, inertial mass is assumed to be approximately equal to the two-floor masses. Therefore, the total kinetic energy of this setup is given by

$$
T=T_{\text {mass } 1_{1}}+T_{\text {mass }_{2}}=\frac{1}{2} m_{1} \dot{x}_{1}^{2}(t)+\frac{1}{2} m_{2} \dot{x}_{2}^{2}(t),
$$

where $x_{1}$ and $x_{2}$ are the displacements of the two inertial masses $\left(m_{1}\right.$ and $\left.m_{2}\right)$ when its base is perturbed by a displacement $z$; also, $\left({ }^{\circ}\right)$ represents the time derivative $\frac{d}{d t}()$. The expression for the potential energy of the host structure can be expressed using

$$
U=\frac{1}{2} k_{1}\left(x_{2}-z\right)^{2}+\frac{1}{2} k_{2}\left(x_{2}-x_{1}\right)^{2},
$$


where $k_{1}$ and $k_{2}$ are the effective stiffnesses of levels 1 and 2 of the two story model, respectively. Each level is modeled as four clamped-guided beams in parallel. Thus, the effective stiffness has the relation

$$
k_{i}=\frac{12 E I}{L_{i}^{3}} N, \quad i=1,2 \text { and } N=4,
$$

where $E$ and $I$ are the Young's modulus and the moment of inertia of the four supporting beams, respectively. Following the Lagrangian approach, the equations of motion of the host structure take the following form

$$
\underbrace{\left[\begin{array}{cc}
m_{1} & 0 \\
0 & m_{2}
\end{array}\right]}_{M}\left\{\begin{array}{l}
\ddot{x}_{1} \\
\ddot{x}_{2}
\end{array}\right\}+\underbrace{\left[\begin{array}{cc}
k_{1} & -k_{1} \\
-k_{1} & k_{1}+k_{2}
\end{array}\right]}_{K}\left\{\begin{array}{l}
x_{1} \\
x_{2}
\end{array}\right\}=\underbrace{\left[\begin{array}{c}
0 \\
k_{2}
\end{array}\right]}_{\mathcal{B}}\{z\},
$$

where $\mathcal{B}$ is the control influence matrix. Analytically, the transfer functions have the form

$$
\frac{X(i \omega)}{Z(i \omega)}=\left(-M \omega^{2}+i C \omega+K\right)^{-1} \mathcal{B}
$$

where the damping matrix $C$ is obtained from the experimental modal damping values following the procedure shown in [11]. However, if the supporting columns also contribute to the mass of the structure, the inertial contribution of the supporting columns should also be included in the mass matrix $(M)$. Therefore, the kinetic energy of the four columns supporting the $i^{\text {th }}$ story is given by

$$
\begin{aligned}
T_{\text {level }_{i}} & =N\left(\frac{1}{2} \rho A \int_{0}^{L_{2}}\left(\alpha_{i} \dot{x}_{i-1}+\beta_{i} \dot{x}_{i}\right)^{2} d y\right), \\
& =\frac{1}{2} \underbrace{N\left[\rho A \int_{0}^{L_{i}} \alpha_{i}^{2} d y\right]}_{m_{i 1}^{b}} \dot{x}_{i-1}^{2}+\underbrace{N\left[\rho A \int_{0}^{L_{i}} \alpha_{i} \beta_{i} d y\right]}_{\mathrm{m}_{i 2}^{b}} \dot{x}_{i-1} \dot{x}_{i}+\frac{1}{2} \underbrace{1\left[\rho A \int_{0}^{L_{i}} \beta_{i}^{2} d y\right]}_{\mathrm{m}_{i 3}^{b}} \dot{x}_{i}^{2}, \\
& =\frac{1}{2} \mathrm{~m}_{i 1}^{b} \dot{x}_{i-1}^{2}+\mathrm{m}_{i 2}^{b} \dot{x}_{i-1} \dot{x}_{i}+\frac{1}{2} \mathrm{~m}_{i 3}^{b} \dot{x}_{i}^{2}, \quad i=1,2
\end{aligned}
$$

where

$$
\alpha_{i}=\left[1-\frac{\left(3 L_{i}-2 y\right) y^{2}}{L_{i}^{3}}\right], \quad \beta_{i}=\left[\frac{\left(3 L_{i}-2 y\right) y^{2}}{L_{i}^{3}}\right] .
$$

The total kinetic energy of the 2-DOF setup is given by

$$
\begin{aligned}
T= & T_{\text {mass }_{1}}+T_{\text {level }_{1}}+T_{\text {mass }_{2}}+T_{\text {level }_{2}}, \\
= & \frac{1}{2} m_{1} \dot{x}_{1}^{2}(t)+\frac{1}{2} \mathfrak{m}_{11}^{b} \dot{z}^{2}+\mathrm{m}_{12}^{b} \dot{z} \dot{x}_{1}+\frac{1}{2} \mathrm{~m}_{13}^{b} \dot{x}_{1}^{2} \\
& \quad+\frac{1}{2} m_{2} \dot{x}_{2}^{2}(t)+\frac{1}{2} \mathrm{~m}_{21}^{b} \dot{x}_{1}^{2}+\mathrm{m}_{22}^{b} \dot{x}_{1} \dot{x}_{2}+\frac{1}{2} \mathrm{~m}_{23}^{b} \dot{x}_{2}^{2} .
\end{aligned}
$$

The expressions for the updated kinetic energy and the potential energy can be substituted into the Lagrange equation to obtain the governing equations of the system. The updated equations of motion of the 
host structure are

$$
\left[\begin{array}{ll}
\mathrm{m}_{11} & \mathrm{~m}_{12} \\
\mathrm{~m}_{21} & \mathrm{~m}_{22}
\end{array}\right]\left\{\begin{array}{l}
\ddot{x}_{1} \\
\ddot{x}_{2}
\end{array}\right\}+\left[\begin{array}{cc}
k_{1} & -k_{1} \\
-k_{1} & k_{1}+k_{2}
\end{array}\right]\left\{\begin{array}{l}
x_{1} \\
x_{2}
\end{array}\right\}=\underbrace{\left[\begin{array}{cc}
0 & 0 \\
k_{2} & -\mathrm{m}_{12}^{b}
\end{array}\right]}_{\mathcal{B}}\left\{\begin{array}{l}
z \\
\ddot{z}
\end{array}\right\},
$$

with $\mathrm{m}_{11}=\mathrm{m}_{11}^{b}+m_{1}+\mathrm{m}_{21}^{b}, \mathrm{~m}_{12}=\mathrm{m}_{21}=\mathrm{m}_{22}^{b}$, and $\mathrm{m}_{22}=\mathrm{m}_{23}^{b}+m_{2}$.

\section{Analytical Model of Subordinate Oscillators Arrays}

Dynamic vibration absorbers (DVAs) are ancillary systems attached to a host structure to absorb undesired vibrations of the structure. Typically, DVAs are modeled as a single degree of freedom linear oscillators, as each DVA is tuned to operate primarily at its fundamental natural frequency. When an array of linear oscillators target a frequency range, then the array is referred to as a Subordinate Oscillator Array (SOA). The performance of the SOA depends primarily on the placement of the fundamental natural frequency of each oscillators in the SOA with respect to that of the host structure.

A lumped-mass model of the SOAs attached to each mass of the 2-DOF system is shown in Figure 1. The equations of motion of an SOA connected to mass $\left(m_{i}\right)$ will take a form of

$$
\begin{aligned}
& M_{1 j} \ddot{x}_{1 j}+C_{1 j} \dot{x}_{1 j}-C_{1 j} \dot{x}_{1}+\boldsymbol{K}_{1 j} x_{1 j}-\boldsymbol{K}_{1 j} x_{1}=0, \\
& M_{2 j} \ddot{x}_{2 j}+C_{2 j} \dot{x}_{2 j}-C_{2 j} \dot{x}_{2}+\boldsymbol{K}_{2 j} x_{2 j}-\boldsymbol{K}_{2 j} x_{2}=0,
\end{aligned}
$$

where $\boldsymbol{x}_{1 j}$ and $\boldsymbol{x}_{2 j}$ represent the vector of displacements of the oscillators in the SOAs associated to the $j^{\text {th }}$ natural frequency that are attached to masses 1,2 , respectively. In the above equations, $\boldsymbol{M}_{i j}, \boldsymbol{C}_{i j}$ and $\boldsymbol{K}_{i j}$ represents mass, modal damping, and stiffness vectors of the individual oscillators attached to the primary mass $i$ and associated with natural frequency $j$, respectively. If $\mathbb{X}=\left\{\boldsymbol{x}_{11}^{T}, \boldsymbol{x}_{12}^{T}, \boldsymbol{x}_{21}^{T}, \boldsymbol{x}_{22}^{T}, x_{1}, x_{2}\right\}^{T}$ denotes the vector of states related to the SOAs, the governing equations can be expressed in the form

$$
\mathbb{M} \ddot{X}+\mathbb{C} \dot{X}+\mathbb{K X}=\mathbb{F}
$$

with

$$
\begin{aligned}
& \mathbb{M}=\left[\begin{array}{ll|ll}
\operatorname{diag}\left(M_{11}\right) & & & \\
& \operatorname{diag}\left(M_{22}\right) & & \\
\hline & & \mathrm{m}_{11} & \mathrm{~m}_{12} \\
\mathrm{~m}_{21} & \mathrm{~m}_{22}
\end{array}\right], \quad \mathbb{F}=\left\{\begin{array}{c}
\mathbf{0} \\
\mathbf{0} \\
0 \\
k_{2} z-\mathrm{m}_{12}^{b} \ddot{z}
\end{array}\right\}, \\
& \mathbb{C}=\left[\begin{array}{cc|cc}
\operatorname{diag}\left(\boldsymbol{C}_{11}\right) & & -\boldsymbol{C}_{11} & \mathbf{0} \\
& \operatorname{diag}\left(\boldsymbol{C}_{22}\right) & \mathbf{0} & -\boldsymbol{C}_{22} \\
\hline-\boldsymbol{C}_{11} & \mathbf{0} & c_{11}+\sum C_{11 n} & c_{12} \\
\mathbf{0} & -\boldsymbol{C}_{22} & c_{21} & c_{22}+\sum C_{22 n}
\end{array}\right], \\
& \mathbb{K}=\left[\begin{array}{cc|cc}
\operatorname{diag}\left(\boldsymbol{K}_{11}\right) & & -\boldsymbol{K}_{11} & \mathbf{0} \\
& \operatorname{diag}\left(\boldsymbol{K}_{22}\right) & \mathbf{0} & -\boldsymbol{K}_{22} \\
\hline-\boldsymbol{K}_{11} & \mathbf{0} & k_{2}+\sum K_{11 n} & -k_{2} \\
\mathbf{0} & -\boldsymbol{K}_{22} & -k_{2} & k_{1}+k_{2}+\sum K_{22 n}
\end{array}\right] .
\end{aligned}
$$

In succeeding sections, dynamic model of the host and the subordinate system are validated through experimental testing. 


\section{Testing, Parameter Estimation and Host Model Validation}

This section validates the host structure model via experiments. In [7], Vignola et al. explain the importance of having precise estimates of natural frequencies of the host structure for accurately designing SOAs. Therefore, before proceeding to the design of SOAs, it is crucial to update (if required) and validate the host structure's model. For this reason, the 2-DOF structure shown in Figure 2 is treated as the host structure whose resonant frequencies are targeted with the dynamic oscillators. In this structure, two wooden blocks are supported by four aluminum posts that are attached to a steel base plate. The dimensions and the blueprint of the host structure are provided in the supplementary documents. Additionally, some of the significant geometric parameters of the host structure are tabulated in Tables 1 and 2.

Table 1: Geometric properties of the host structure

\begin{tabular}{ccc}
\hline Parameter & Measured & Tuned \\
\hline \hline & $0.259 \mathrm{~kg}$ & \\
Mass of first block $\left(m_{1}\right)$ & $0.258 \mathrm{~kg}$ & \\
Mass of second block $\left(m_{2}\right)$ & $0.5 \mathrm{in}$ & \\
Width of Al columns & $1 / 16 \mathrm{in}$ & \\
Height of Al columns & $\sim 26.035 \pm 0.635 \mathrm{~cm} \quad(\sim 10.25 \pm 0.25 \mathrm{in})$ & $26.48 \mathrm{~cm}$ \\
Dist. b/w base and $m_{2}$ & $\sim 26.035 \pm 0.635 \mathrm{~cm} \quad(\sim 10.25 \pm 0.25 \mathrm{in})$ & $25.62 \mathrm{~cm}$ \\
Dist. b/w $m_{1}$ and $m_{2}$ & $\sim$ & \\
\hline
\end{tabular}

Table 2: Material and dynamic properties of the host structure

\begin{tabular}{ccccc}
\hline Parameter & Estimates & Tuned & Experimental & Error \\
\hline \hline & & & & \\
E of Aluminum & $69 \mathrm{GPa}$ & & & \\
$\rho$ of Aluminum & $2700 \mathrm{~kg} / \mathrm{m}^{3}$ & & & \\
& & & & \\
First natural frequency & $4.94 \mathrm{~Hz}$ & $4.76 \mathrm{~Hz}$ & $4.76 \mathrm{~Hz}$ & \\
Second natural frequency & $12.99 \mathrm{~Hz}$ & $12.87 \mathrm{~Hz}$ & $12.87 \mathrm{~Hz}$ & \\
\hline
\end{tabular}

Experimental frequency response functions (FRFs) are measured by exciting the base of the structure with an APS (Electro-SEIS 113) shaker, as shown in Figure 2. With accelerometers attached to the base plate and the two wooden blocks, an LMS SCADAS Mobile data acquisition system was used to measure the two transmissibility transfer functions. Subsequently, the Polymax algorithm was used to determine the natural frequencies and the corresponding modal damping ratios from the measured FRFs. The dynamic model discussed in the previous section was compared with the experimental results. Then, the column lengths in the model were updated so that the model represents the experiment more accurately.

Engineering judgment plays a vital role in updating the model by selecting parameters that are the sources of uncertainty. Joints add significant complexity in representing the dynamics of the structure as a lumped-mass system. For instance, if any one of the supporting columns is not rigidly secured, its stiffness contribution is reduced; as a result, experiments yield lower results of the host's natural frequencies. The other source of uncertainty comes from the effective length of the supporting column between any two levels. Although the length of the support structure affects both the mass and the stiffness matrices, its contribution to the stiffness matrix is dominant. This is due to the cubic relationship in Eq. 3. 


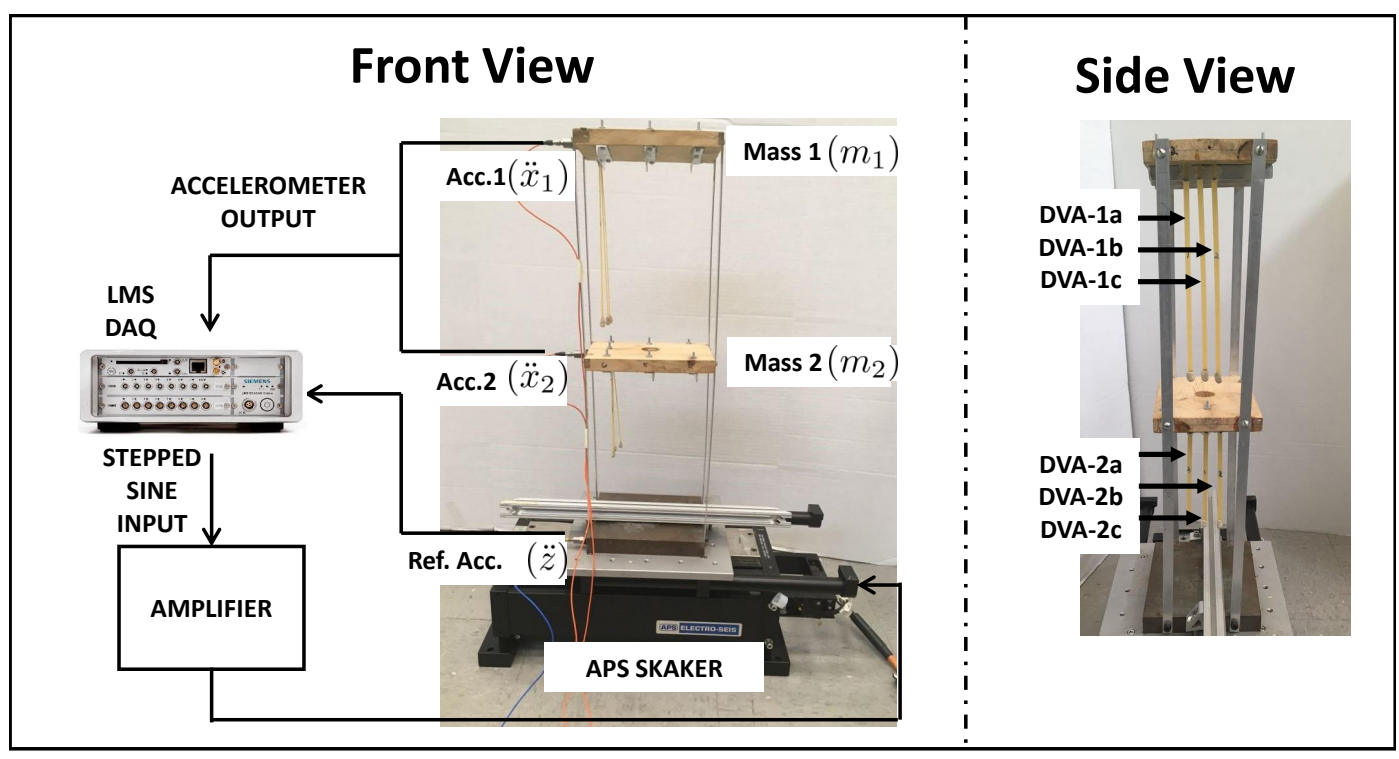

Figure 2: Host Structure Setup

In a classroom, such uncertainties in the experimental setup provide an opportunity for students to develop intuition through trial and error. In the experimental setup, the mass of the two wooden blocks and the cross-sectional dimensions of the beam can be measured. However, in the current setup, the uncertainty in measuring the effective length of the supporting posts comes from the thickness of the two wooden blocks attached to it. Therefore, the length of the posts is one parameter that is updated in the model by defining a cost function in terms of experimental and simulated natural frequencies:

$$
J_{1}=\left\|\omega_{\text {exp }, 1}-\omega_{\text {model }, 1}\left(L_{1}, L_{2}\right)\right\|_{2}+\left\|\omega_{\text {exp }, 2}-\omega_{\text {model }, 2}\left(L_{1}, L_{2}\right)\right\|_{2} .
$$

The column lengths $L_{1}$ and $L_{2}$ that minimize the above cost function are the effective lengths of the posts. An iterative approach is adopted to estimate the optimal lengths by searching in the range of uncertainty. Finally, the updated properties of the host structure are tabulated in Table 1. Also, the modal damping estimates used in the simulations are $0.2 \%$ and $0.35 \%$ for the first and the second models, respectively. Table 2 summarizes the characteristics of the dynamic model before and after updating the equivalent stiffness and modal damping.

\section{Testing, Parameter Estimation and SOA Design}

This section discusses the design of SOAs to attenuate the dynamics of the 2-DOF structure shown in Figure 2. These SOAs are developed using fettuccine strands and modeling clay. While the cantilevered fettuccine strand predominately contributes to the stiffness of each oscillator, the modeling clay attached to its tip allows for fine-tuning of its fundamental frequency. In a classroom, fettuccine and modeling clay are excellent and affordable candidates for teaching the concepts of vibration absorption as they capture students' attention and keep them engaged throughout the experimentation. As the chosen materials are unconventional candidates, the material properties are unknown and non-homogeneous, and therefore, the analysis of the system has multiple levels of complexity. Additionally, the variation in the dynamic properties between fettuccine strands gives students an opportunity to deal with uncertainty.

Once the nominal material properties are estimated, the next step is to design an oscillator tuned to a particular frequency. If an oscillator is tuned to the natural frequency of a host structure, the corresponding peak in the frequency response function (FRF) splits into two newer peaks, and an anti-resonant 

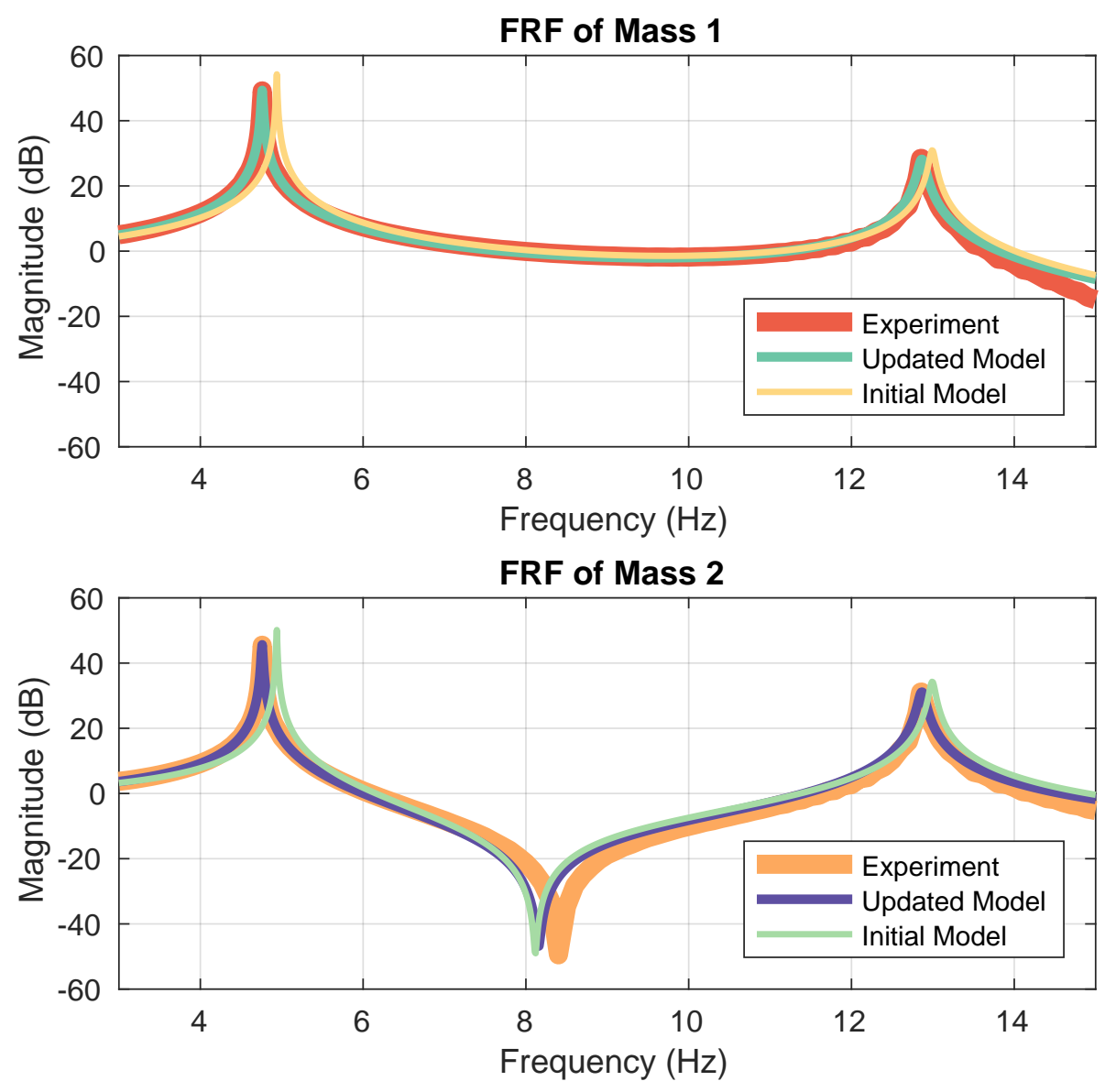

Figure 3: Experimental and simulated FRFs of the host structure.

valley appears at the tuned frequency. A DVA is an example of such an oscillator which, when tuned to the host's natural frequency, absorbs the host's energy and creates a new FRF with two peaks. However, the dynamics of the system can deteriorate when the modified structure is under broadband excitation, thereby activating the two newer resonant peaks. As a result, the natural progression of this discussion seeks an array of oscillators (SOAs) that attenuates the dynamic response over a broader frequency bandwidth.

Designing and implementing SOAs using fettuccine strands in a classroom is very engaging as it is highly intuitive to tune oscillators. However, students would realize that for precise tuning of each oscillator, it is necessary to estimate the accurate dynamic properties of individual fettuccine strands. In this regard, towards the end of this section, an iterative process is proposed to develop better performing SOAs under the presence of uncertainties. The rest of this section provides details of each of these steps along with corresponding experimental results.

\subsection{Estimation of material properties of Fettuccine strands}

In this section, the average Young's modulus of oscillators is experimentally estimated using the dynamics test setup shown in Figure 4b. The tip response of the cantilevered fettuccine strand (without any tip-mass) is measured with a PSV-100 laser vibrometer as the base is mounted to an APS shaker. The resonant frequency of this strand is then determined from the experimental FRF. The Young's modulus of the fettuccine strands are estimated from the measured resonant frequency $(\omega)$ and the average material 
density $(\rho)$ using the analytical relationship [11] given by

$$
\omega=\sqrt{\frac{3 E I}{(0.24 \rho A L) L^{3}}},
$$

where the geometric parameters are length $(L)$, cross-sectional area $(A)$ and moment-of-inertia $(I)$. Table 3 presents the average structural parameters of fettuccine strands. Figure 4a shows the frequency response function of a fettuccine strand as its length is varied, and Table 3 lists the corresponding natural frequencies and the damping ratios. Based on these experimental results, one can see that Young's modulus of the fettuccine strand varies from $2.14 \times 10^{9} \mathrm{~Pa}$ to $2.63 \times 10^{9} \mathrm{~Pa}$. Since the other parameters do not show as significant a variation as the Young's Modulus, uncertainties in the FRFs can be primarily attributed to the variance in Young's modulus.

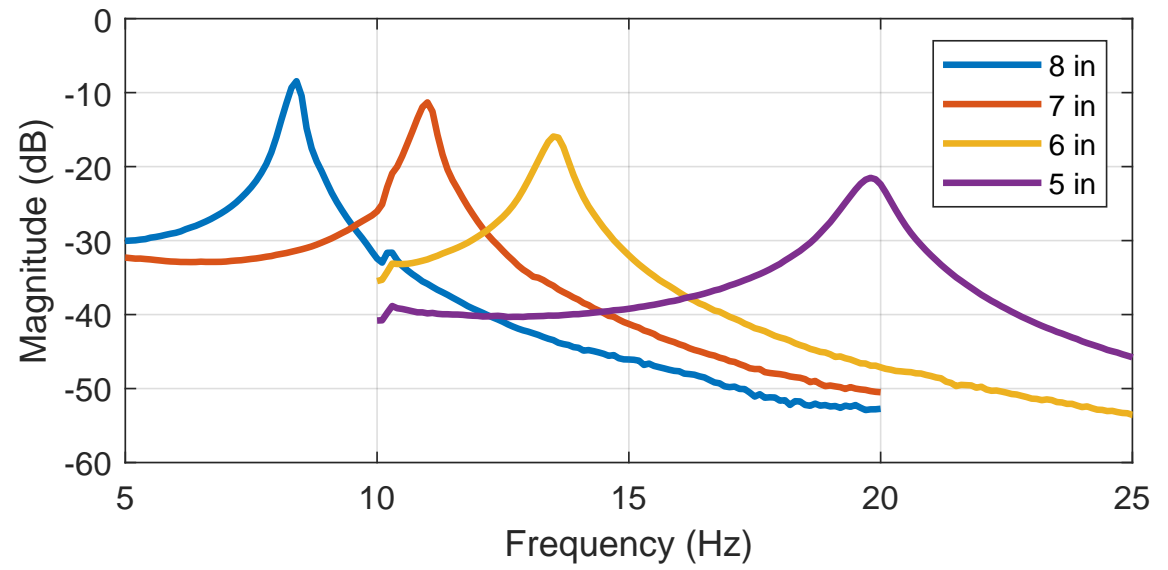

(a)

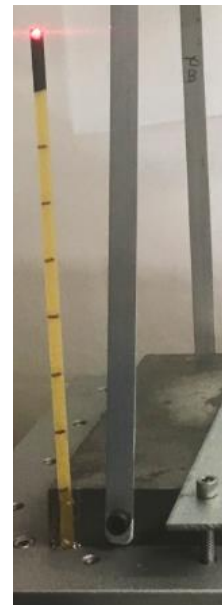

(b)

Figure 4: (a) Experimental FRFs of fettuccine strands of varying lengths (Input: acceleration of the base, Output: Tip-velocity of the fettuccine strand), (b) Dynamic test of a single cantilevered fettuccine strand

Table 3: Measured and estimated physical parameters of fettuccine strands

\begin{tabular}{ccc|c||c|c}
\hline DVA length & Exp. $\eta$ & Exp. $\zeta$ & Estimated $E$ & Parameter & Value \\
\hline \hline 8 in & $8.35045 \mathrm{~Hz}$ & $1.76494 \%$ & $2.5851 \mathrm{GPa}$ & Average Thickness & $1.4 \mathrm{~mm}$ \\
$7 \mathrm{in}$ & $11.0095 \mathrm{~Hz}$ & $1.8529 \%$ & $2.634 \mathrm{GPa}$ & Average Width & $5.6 \mathrm{~mm}$ \\
$6 \mathrm{in}$ & $13.5171 \mathrm{~Hz}$ & $1.89777 \%$ & $2.1432 \mathrm{GPa}$ & Average Density & $1115.8 \mathrm{~kg} / \mathrm{m}^{3}$ \\
$5 \mathrm{in}$ & $19.7543 \mathrm{~Hz}$ & $2.31913 \%$ & $2.2075 \mathrm{GPa}$ & Area Moment of Inertia & $1.29 e-12 \mathrm{~m}^{4}$ \\
\hline \multicolumn{5}{r}{} \\
\hline
\end{tabular}

$\mu$ is mean estimate, $\eta$ - natural frequency, $\zeta$ - modal damping ratio and $E$ - Young's modulus

\subsection{Numerical simulation of SOAs}

Before proceeding to the experimental implementation of SOAs, the dynamics of the SOAs are simulated using an array of identical fettuccine strands with Young's modulus and modal damping ratio equal to the experimental mean estimate of $2.39 \mathrm{GPa}$ and $1.95 \%$, respectively. The other parameters of the oscillators are presented in Table 4. 

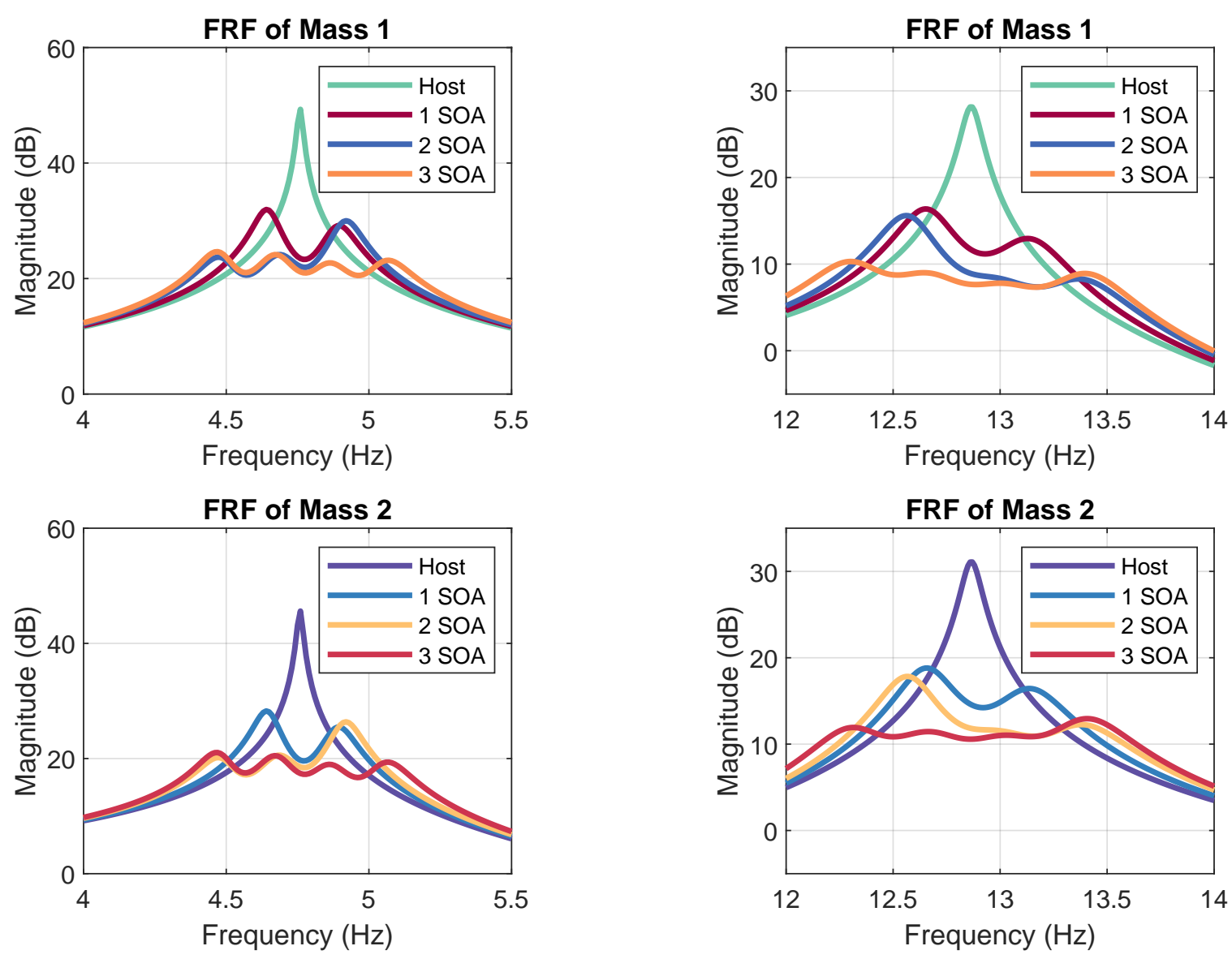

(a)

(b)

Figure 5: Simulated FRFs of the host structure as oscillators are added to build the SOA at (a) the first resonant peak, and (b) the second resonant peak

Figure 5 shows the FRF of the host structure with and without oscillators. As oscillators are added one at a time, the magnitude of the FRF is reducing over a broader frequency bandwidth. SOAs with three oscillators is able to flatten the FRF over the frequency bandwidth of interest. Typically, a tuned oscillator targets the peak frequency of the system. However, sometimes, due to practical limitations, they are mistuned, i.e., the peak frequency and the target frequency are not equal to each other. Table 4 presents the selected target and peak frequencies of all oscillators in the two SOAs. The peak's magnitude and the vibration attenuation achieved in each iteration is also shown in Figure 5. After addition of three oscillators, the (minimum) vibration attenuation achieved are approximately $25 d B$ and $18 d B$ at the first and second natural frequency bands, respectively. Figure 5 also shows that the addition of SOAs has resulted in modal spillover. In other words, there is a minimal increase in magnitude at some of the natural frequencies around the host's initial peak. However, this increase in magnitude is negligible when compared to the extent of attenuation achieved by using SOAs.

As seen in Figure 5a, oscillator OSC-1a tuned to peak frequency splits the resonant peak into two peaks of equal magnitudes. However, in Figure $5 \mathrm{~b}$ oscillator OSC-2a is mistuned and as a result, it splits the resonant peak into two peaks of differing magnitudes. Similar behavior is observed with other oscillators in the two SOAs. It is also interesting to note that with the current approach targeting peak frequencies, even number of oscillators or mistuned oscillators in the SOA results in FRFs that have 
Table 4: Peak magnitude and attenuation achieved by SOAs as observed in numerical simulations.

\begin{tabular}{c|l|cc|cc|cc}
\hline \multirow{2}{*}{$\begin{array}{c}\text { Natural Freq. } \\
(\text { SOA })\end{array}$} & \multirow{2}{*}{ Oscillator (OSC) } & \multicolumn{2}{|c|}{ Frequency } & \multicolumn{2}{|c}{ Max Peak Magnitude } & \multicolumn{2}{c}{ Max Peak Attenuation } \\
& & Max Peak & Target & Mass 1 & Mass 2 & Mass 1 & Mass 2 \\
\hline \hline \multirow{2}{*}{$1^{s t}$} & No OSC & $4.76 ~ H z$ & $4.76 ~ H z$ & $45.6 d B$ & $49.29 d B$ & - & - \\
& OSC - 1a & $4.92 ~ H z$ & $4.92 ~ H z$ & $28.25 d B$ & $31.95 d B$ & $17.35 d B$ & $17.34 d B$ \\
$($ SOA-1) & OSC - 1a, 1b & $4.56 ~ H z$ & $4.58 ~ H z$ & $26.37 d B$ & $30.01 d B$ & $19.23 d B$ & $19.28 d B$ \\
& OSC - 1a, 1b, 1c & $4.65 H z$ & - & $21.03 d B$ & $24.65 d B$ & $24.57 d B$ & $24.64 d B$ \\
\hline \multirow{2}{*}{$2^{\text {nd }}$} & No OSC & $12.86 ~ H z$ & $12.76 ~ H z$ & $31.09 d B$ & $28.14 d B$ & - & - \\
& OSC - 2a & $12.5 ~ H z$ & $12.55 ~ H z$ & $18.81 d B$ & $16.37 d B$ & $12.28 d B$ & $11.77 d B$ \\
$($ SOA-2) & OSC - 2a, 2b & $13.20 ~ H z$ & $13.20 ~ H z$ & $17.84 d B$ & $15.6 d B$ & $13.25 d B$ & $12.54 d B$ \\
& OSC - 2a, 2b, 2c & $12.2 ~ H z$ & - & $12.95 d B$ & $10.3 d B$ & $18.14 d B$ & $17.84 d B$ \\
\hline
\end{tabular}

uneven peak magnitudes. Thus, the question arises whether SOAs can be implemented practically and the next subsection discusses this question using experimental results.

\subsection{Designing SOAs using oscillators with uncertain material properties}

This section studies the performance of SOAs when the variation in Young's modulus of the fettuccine strands is neglected and oscillators based on nominal material properties estimated in the previous section are used to construct the SOAs. Fettuccine based oscillators are added to the host structure one at a time, and the tip-mass is calculated using the nominal Young's modulus to tune the oscillator to the peak frequency. However, due to the uncertainty, the oscillator's natural frequency may not match the target frequency. Rather than updating the tip-mass, a new oscillator is added, and the process is repeated until a flat FRF is achieved. The details of the tip-masses, the lengths of the fettuccine stands and the target frequencies $(\eta)$ are tabulated for the two SOAs in Table 5. Eventually, two SOAs of nine and six oscillators resulted in a flat FRF at the first and the second resonant frequencies of the host structure. SOA-1 and SOA-2 are attached to the top and the bottom wooden blocks, respectively as shown in Figure 6b. Figure 6a shows the FRF of the modified structure with SOAs. This figure shows that with SOAs, the dynamic response of the host structure can be attenuated over a broad frequency bandwidth in spite of uncertain material properties. However, the attenuated response is uneven with a nonuniform magnitude in its frequency bandwidth. If precise dynamic properties of the oscillators are known, the resulting attenuated response will result in uniform magnitude. Also, a comparable degree of attenuation could be achieved with a fewer number of oscillators. In the next section, an iterative approach is proposed to update fettuccine's material properties, thereby reducing the uncertainties while designing SOAs.

\subsection{Designing SOAs using oscillators by updating material properties}

The flowchart in Figure 7 presents a generic approach to design SOAs in the presence of uncertainties. The design approach is primarily based on the idea of estimating the natural frequency of a substructure after it is attached to a host/primary structure. In this paper, the analysis is restricted to a simplified method when the approximate range of variation of the parameter is known. From preliminary experiments conducted, it is observed that the uncertainty in the substructure arose primarily due to variance in Young's modulus of the material. The approximate variation of the Young's Modulus assessed in previous sections is used here. Furthermore, this method inherently assumes that the mass of each subordinate oscillator is negligible when compared to that of the host structure.

The first step in the design process involves the selection of a frequency band with a resonant peak. Then a single oscillator is attached such that its natural frequency is higher than the selected frequency 

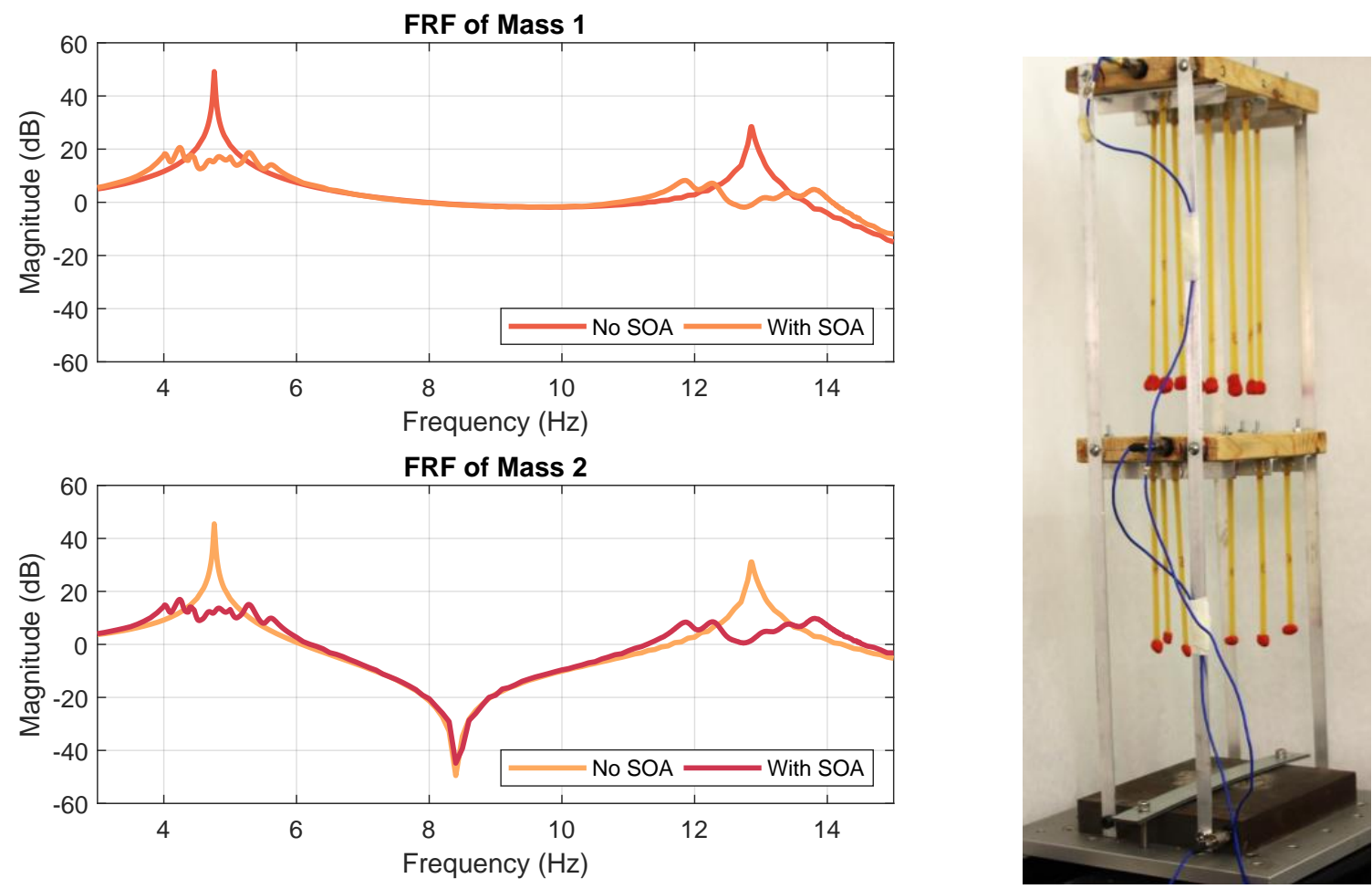

(a)

(b)

Figure 6: Vibration attenuation using SOAs made of oscillators with unknown material properties

Table 5: Properties and target frequencies of the oscillators with uncertain Young's modulus that achieved flat band attenuation of the host

\begin{tabular}{c|ccc|ccc|cc|c}
\hline & \multicolumn{7}{|c}{ SOA-1 } \\
\hline Oscillator & OSC-1a & OSC-1b & OSC-1c & OSC-1d & OSC-1e & OSC-1f & OSC-1h & OSC-1h & OSC-1i \\
Length & $8 \mathrm{in}$ & $8 \mathrm{in}$ & $8 \mathrm{in}$ & $8 \mathrm{in}$ & $8 \mathrm{in}$ & $8 \mathrm{in}$ & $8 \mathrm{in}$ & $8 \mathrm{in}$ & $8 \mathrm{in}$ \\
Tip-mass & $0.80 \mathrm{~g}$ & $1.00 \mathrm{~g}$ & $1.03 \mathrm{~g}$ & $0.83 \mathrm{~g}$ & $1.27 \mathrm{~g}$ & $1.35 \mathrm{~g}$ & $1.36 \mathrm{~g}$ & $0.71 \mathrm{~g}$ & $0.65 \mathrm{~g}$ \\
Target $\eta$ & $4.78 \mathrm{~Hz}$ & $4.70 \mathrm{~Hz}$ & $4.60 \mathrm{~Hz}$ & $5.04 \mathrm{~Hz}$ & $4.47 \mathrm{~Hz}$ & $4.39 \mathrm{~Hz}$ & $4.4 \mathrm{~Hz}$ & $5.21 \mathrm{~Hz}$ & $5.3 \mathrm{~Hz}$ \\
\hline & \multicolumn{7}{|c}{ SOA-2 } \\
\hline Oscillator & OSC-2a & OSC-2b & OSC-2c & OSC-2d & OSC-2e & OSC-2f & & \\
Length & $5 \mathrm{in}$ & $5 \mathrm{in}$ & $5 \mathrm{in}$ & $5 \mathrm{in}$ & $5 \mathrm{in}$ & $5 \mathrm{in}$ & & \\
Target $\eta$ & $12.98 \mathrm{~Hz}$ & $13.14 \mathrm{~Hz}$ & $12.47 \mathrm{~Hz}$ & $12.31 \mathrm{~Hz}$ & $13.53 \mathrm{~Hz}$ & $12.17 \mathrm{~Hz}$ & & \\
\hline
\end{tabular}

band. The assumptions mentioned earlier become relevant in this step. After attaching the fettuccine strand (without tip-mass) to the host structure, the effective natural frequency of the substructure can be estimated by placing an accelerometer at the base of the oscillator and measuring its tip response. Even 


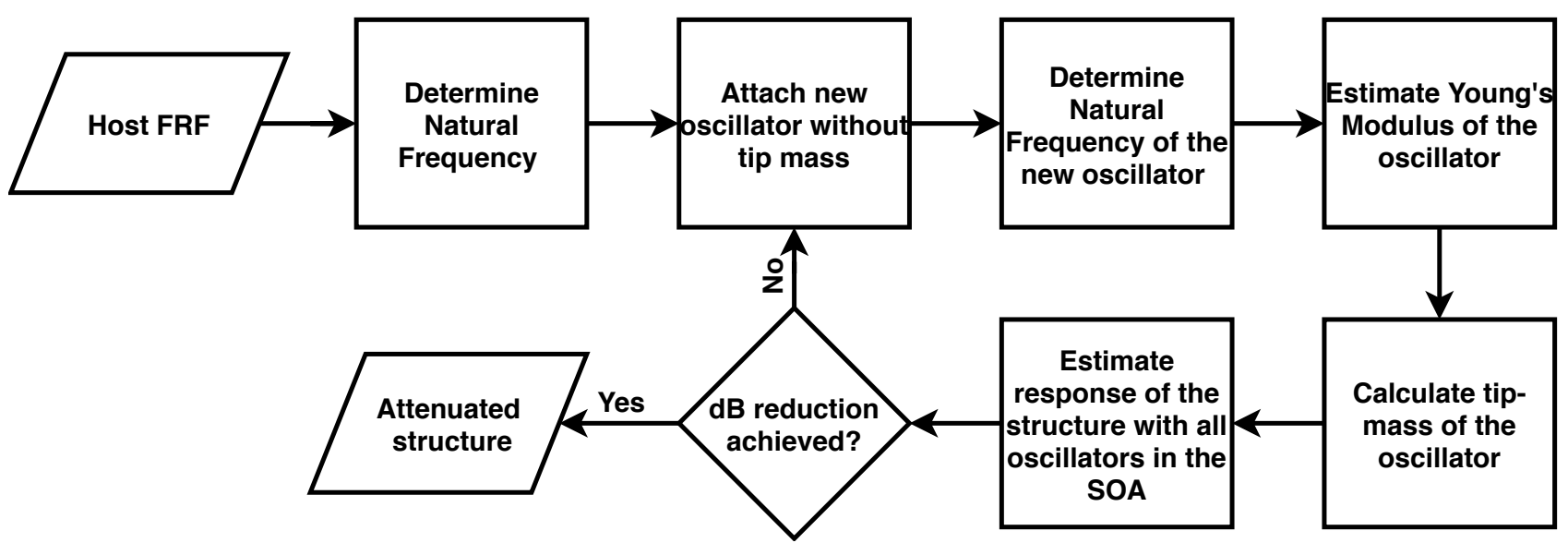

Figure 7: Flow chart of the iterative procedure followed to estimate material properties of fettuccine strands and therefore design oscillators tuned to the desired target frequency.

though the substructure is attached to the host, the natural frequency of the substructure can be identified from the frequency response of the substructure measured between its base and its tip. However, in cases where the dynamics of the oscillator cannot be directly measured, the FRF between the excitation and the response of the host structure is sufficient. For simple structures such as the one under study, care is taken such that the natural frequency of the fettuccine strands (without tip-mass) is sufficiently away from the two frequency bandwidth of interest. In such a scenario, a new peak is introduced in the frequency response function of the host structure. For instance, an untuned fettuccine strand of 8 in has natural frequency around $8.5 \mathrm{~Hz}$, which does not lie in the two frequency bandwidths of interest [ 4-5.5] $\mathrm{Hz}$ and [ 12-14 ] $\mathrm{Hz}$.

Due to the low mass-ratio as well the fact that the natural frequency of the substructure is significantly different from that of the host, the frequency corresponding to the induced peak can be considered as a good approximation of the substructure's natural frequency. This is then tuned to target the peak at 4.76 $\mathrm{Hz}$ by attaching a calculated tip-mass of $0.914 \mathrm{~g}$. Table 4 has further details of all oscillators, where the natural frequencies of the oscillators are estimated using Polymax algorithm. A precise estimate of Young's modulus of each fettuccine strand is calculated using Eq. 11.

Table 6: Estimated dynamic characteristics of the individual oscillators

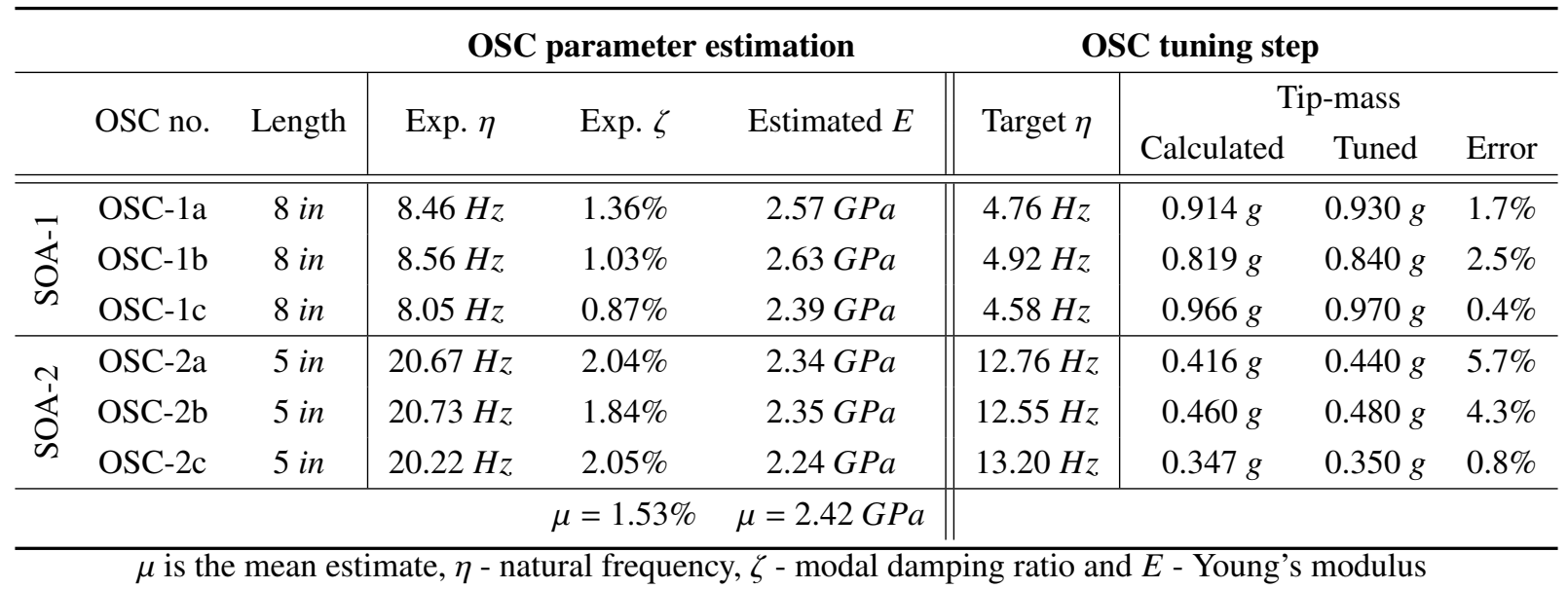

Once the precise flexural stiffness is estimated, the natural step is to determine the tip-mass to tune the oscillator. Previously, the tip-mass is estimated based on the peaks in the FRFs. The magnitudes of 

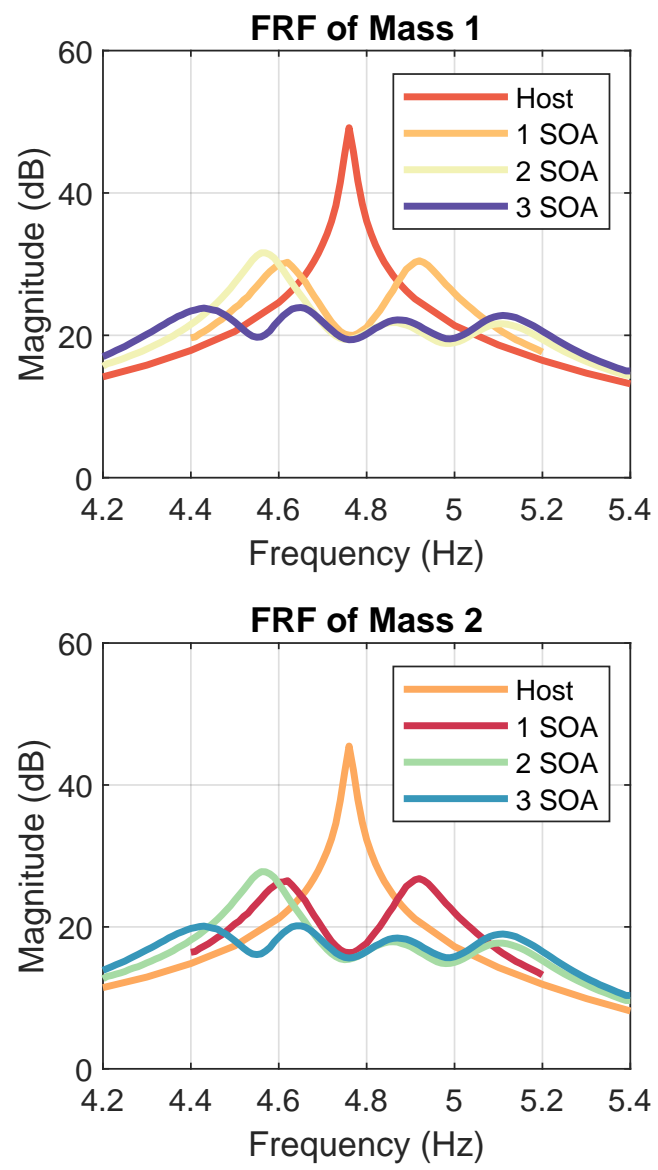

(a)
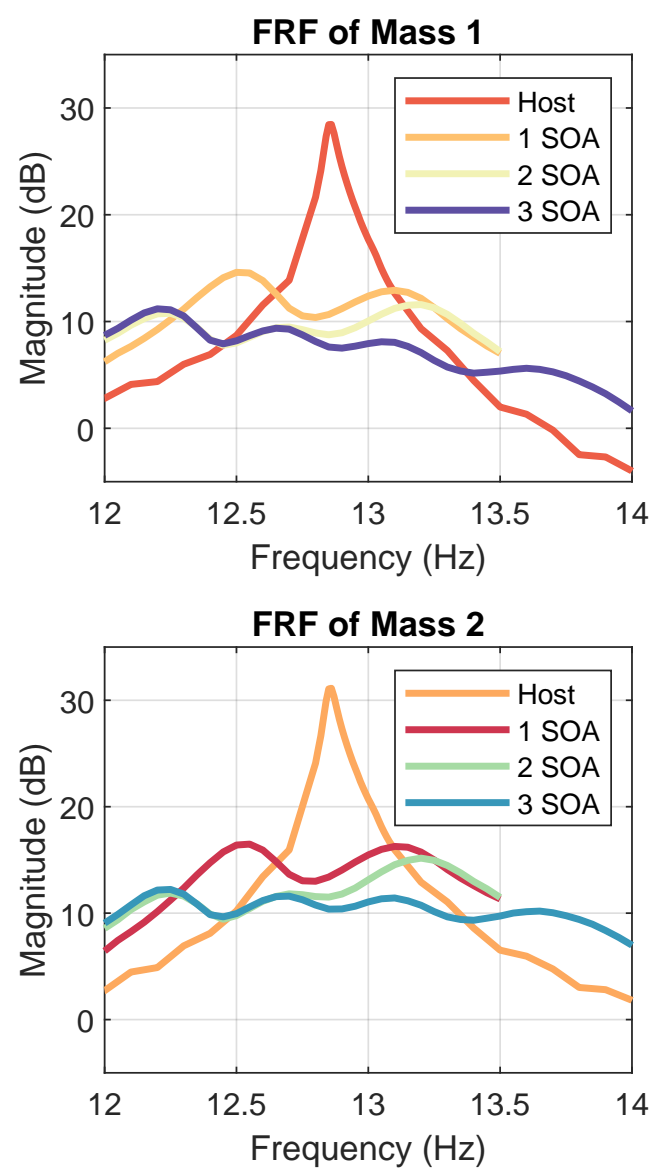

(b)

Figure 8: Experimental Iterative FRFs of SOAs

Table 7: Peak magnitude and attenuation achieved during each step of iterative SOA design while following the proposed design methodology

\begin{tabular}{|c|c|c|c|c|c|c|c|}
\hline \multirow{2}{*}{ Natural Freq. } & \multirow{2}{*}{ OSC-No. } & \multicolumn{2}{|c|}{ Frequency } & \multicolumn{2}{|c|}{ Peak's Magnitude } & \multicolumn{2}{|c|}{ Attenuation } \\
\hline & & Peak's & Target's & Mass 1 & Mass 2 & Mass 1 & Mass 2 \\
\hline \multirow{4}{*}{$1^{s t}$} & No OSC & $4.76 \mathrm{~Hz}$ & $4.76 \mathrm{~Hz}$ & $49.17 d B$ & $45.48 d B$ & - & - \\
\hline & OSC - 1a & $4.92 \mathrm{~Hz}$ & $4.92 \mathrm{~Hz}$ & $30.47 d B$ & $26.81 d B$ & $18.6 \mathrm{~dB}$ & $18.67 d B$ \\
\hline & OSC $-1 a, 1 b$ & $4.56 \mathrm{~Hz}$ & $4.58 \mathrm{~Hz}$ & $31.59 \mathrm{~dB}$ & $27.79 d B$ & $17.58 \mathrm{~dB}$ & $17.69 d B$ \\
\hline & OSC - 1a, 1b, 1c & $4.65 \mathrm{~Hz}$ & - & $23.91 d B$ & $20.15 d B$ & $25.26 d B$ & $25.33 d B$ \\
\hline \multirow{4}{*}{$2^{\text {nd }}$} & No OSC & $12.86 \mathrm{~Hz}$ & $12.76 \mathrm{~Hz}$ & $28.46 d B$ & $31.13 d B$ & - & - \\
\hline & OSC $-2 \mathrm{a}$ & $12.5 \mathrm{~Hz}$ & $12.55 \mathrm{~Hz}$ & $14.61 \mathrm{~dB}$ & $16.49 d B$ & $13.85 d B$ & $14.64 d B$ \\
\hline & OSC $-2 a, 2 b$ & $13.20 \mathrm{~Hz}$ & $13.20 \mathrm{~Hz}$ & $11.59 \mathrm{~dB}$ & $15.17 d B$ & $16.87 d B$ & $15.96 \mathrm{~dB}$ \\
\hline & OSC $-2 \mathrm{a}, 2 \mathrm{~b}, 2 \mathrm{c}$ & $12.2 \mathrm{~Hz}$ & - & $11.19 \mathrm{~dB}$ & $12.23 \mathrm{~dB}$ & $17.27 d B$ & $18.9 \mathrm{~dB}$ \\
\hline
\end{tabular}

the two new peaks may not be equal, as natural frequencies of the oscillators in an SOA are very close to each other. Alternatively, the tip-mass is estimated such that the target peak splits into two peaks of equal magnitudes. The analytical model of the 2-DOF system with oscillators is used to iterate through a set of 

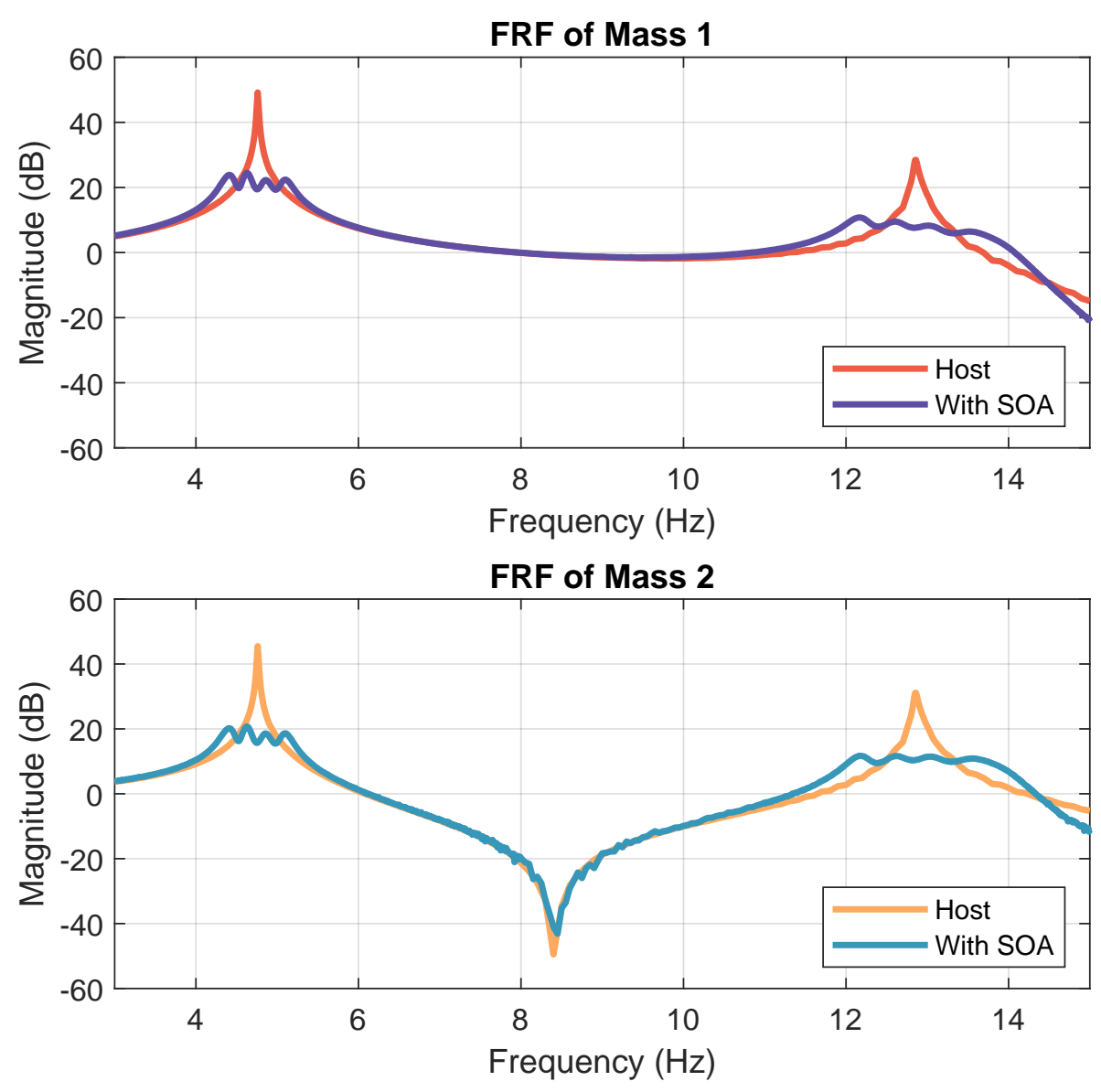

Figure 9: Complete experimental FRF of the host with SOAs made of fettuccine and modeling clay

permissible tip-masses. Consequently, the tip-mass resulting in two peaks of equal magnitudes is added to the oscillator. The above procedure is repeated until the desired reduction in magnitude, or flat FRF is achieved.

This design methodology is implemented on the current test-setup, and the corresponding experimental results are presented in Figure 8. As discussed earlier, the fettuccine oscillators are first attached (without tip-mass) to the host to estimate its modal damping and Young's modulus. Table 6 summarizes these estimates along with the tip-mass calculated from simulations. This table also compares the calculated tip-mass value with the final attached clay mass that resulted in peaks of equal magnitude in the FRF. This discrepancy in the theoretical and the actual tip-mass values arises because a point mass does not accurately model the clay at the tip of the cantilevered strand.

Table 7 summarizes the peak magnitudes of the modified structure along with the attenuation achieved at each step of the iteration. Additionally, Figure 6 shows the progression of the FRFs as the host is modified. This figure shows that tuning SOAs of three oscillators can experimentally create a flat spectrum similar to the simulation results shown in Figure 5. Also, having knowledge of the precise dynamic properties of the oscillators is important to get reasonably uniform peaks in the frequency bandwidth of interest.

In the previous section, $28.55 \mathrm{~dB}$ and $20.24 \mathrm{~dB}$ reduction in magnitude of the mass 1 's (first and second) natural frequencies were achieved with SOAs of nine and six mistuned oscillators, respectively. However, by precisely tuning the oscillators, $25.26 \mathrm{~dB}$ and $17.27 \mathrm{~dB}$ reduction is achieved for the same 
with two SOAs of three oscillators only. The difference in the quality of the final response is evident from Figure $6 \mathrm{a}$ and Figure 9. Table 8 summarizes the minimum magnitude reduction achieved around the first and second natural frequencies for the 2-DOF system. Also, when a single oscillator case is compared against SOAs, SOAs show a better broadband performance than a single oscillator. The table also indicates that the effectiveness of the SOA designed using random placement methods is only marginally better than that of the SOA designed using the proposed approach. This difference can be attributed to the much higher number of oscillators in the SOA designed using random placement method. One can argue the validity of the comparisons between the SOAs and the single oscillator since their mass ratios differ vastly. This shows that mass-ratio is one of the important parameters, and in the next section SOAs are compared against a single oscillator with matching mass-ratio.

Table 8: Comparison of performance of single oscillator and SOAs designed using random placement and proposed design methods

\begin{tabular}{c|c|cc|c}
\hline \multirow{2}{*}{ Natural Freq. } & Absorber & \multicolumn{2}{|c|}{ Magnitude Reduction across Band } & Mass Ratio \\
& & Mass 1 & Mass 2 & \\
\hline \hline \multirow{3}{*}{$1^{\text {st }}$} & Single oscillator (OSC-1a) & $18.6 d B$ & $18.67 d B$ & $0.4 \%$ \\
& SOA - random placement & $28.55 d B$ & $28.5 d B$ & $3.9 \%$ \\
& SOA - proposed method & $25.26 d B$ & $25.33 d B$ & $1.2 \%$ \\
\hline \multirow{2}{*}{$2^{\text {nd }}$} & Single oscillator (OSC-2a) & $13.85 d B$ & $14.64 d B$ & $0.2 \%$ \\
& SOA - random placement & $20.239 d B$ & $21.325 d B$ & $1.5 \%$ \\
& SOA - proposed method & $17.27 d B$ & $18.9 d B$ & $0.7 \%$ \\
\hline
\end{tabular}

Table 9: Magnitude reduction at peak's frequency at different damping ratios of the oscillators in the SOA

\begin{tabular}{|c|c|c|c|c|c|c|c|}
\hline \multirow{2}{*}{ Natural Freq. } & \multirow{2}{*}{ Damping Ratio } & \multicolumn{2}{|c|}{ Peak Magnitude } & \multicolumn{2}{|c|}{ Peak Frequency } & \multicolumn{2}{|c|}{ Reduction at Peak's Frequency } \\
\hline & & Mass 1 & Mass 2 & Mass 1 & Mass 2 & Mass 1 & Mass 2 \\
\hline \multirow{6}{*}{$1^{s t}$} & Host & $49.17 d B$ & $45.48 d B$ & $4.76 \mathrm{~Hz}$ & $4.76 \mathrm{~Hz}$ & - & - \\
\hline & $0.1 \%$ & $42.78 d B$ & $38.86 d B$ & $4.471 \mathrm{~Hz}$ & $4.471 \mathrm{~Hz}$ & $6.39 d B$ & $6.62 d B$ \\
\hline & $0.5 \%$ & $32.96 \mathrm{~dB}$ & $29.1 d B$ & $4.469 \mathrm{~Hz}$ & $4.469 \mathrm{~Hz}$ & $16.21 d B$ & $16.38 d B$ \\
\hline & $1 \%$ & $28.39 d B$ & $24.63 d B$ & $4.466 \mathrm{~Hz}$ & $4.466 \mathrm{~Hz}$ & $20.78 d B$ & $20.85 d B$ \\
\hline & $1.96 \%$ & $24.86 d B$ & $21.25 d B$ & $4.466 \mathrm{~Hz}$ & $4.466 \mathrm{~Hz}$ & $24.31 d B$ & $24.23 d B$ \\
\hline & $3 \%$ & $23.99 d B$ & $20.35 d B$ & $4.677 \mathrm{~Hz}$ & $4.668 \mathrm{~Hz}$ & $25.18 d B$ & $25.13 d B$ \\
\hline \multirow{6}{*}{$2^{\text {nd }}$} & Host & $28.46 d B$ & $31.13 d B$ & $12.86 \mathrm{~Hz}$ & $12.86 \mathrm{~Hz}$ & - & - \\
\hline & $0.1 \%$ & $24.34 d B$ & $27.48 d B$ & $12.3 \mathrm{~Hz}$ & $13.41 \mathrm{~Hz}$ & $4.12 d B$ & $3.65 d B$ \\
\hline & $0.5 \%$ & $16.53 \mathrm{~dB}$ & $19.98 d B$ & $12.29 \mathrm{~Hz}$ & $13.42 \mathrm{~Hz}$ & $11.93 \mathrm{~dB}$ & $11.15 d B$ \\
\hline & $1 \%$ & $12.87 \mathrm{~dB}$ & $16.04 d B$ & $12.28 \mathrm{~Hz}$ & $13.43 \mathrm{~Hz}$ & $15.59 \mathrm{~dB}$ & $15.09 d B$ \\
\hline & $1.96 \%$ & $10.3 d B$ & $12.94 d B$ & $12.3 \mathrm{~Hz}$ & $13.42 \mathrm{~Hz}$ & $18.16 d B$ & $18.19 d B$ \\
\hline & $3 \%$ & $9.874 d B$ & $12.3 \mathrm{~dB}$ & $12.59 \mathrm{~Hz}$ & $12.69 \mathrm{~Hz}$ & $18.586 d B$ & $18.83 d B$ \\
\hline
\end{tabular}

\section{Parametric Study}

\subsection{Effect of damping}

One of the crucial factors that define the flatness of the frequency band is damping of the subordinate oscillators. As evident from Figure 10, the peaks, and valleys in the frequency band disappear as the damping ratio reaches $1.96 \%$, and thus a flatter frequency response is achieved. Note that the value 
$1.96 \%$ is the average damping ratio obtained from the preliminary fettuccine experiments. Table 9 lists the amount of magnitude reduction achieved at different damping ratios. As evident from the figure and the table, increasing the damping ratio reduces the magnitude peaks across the band. However, beyond a critical damping value, which is $1.96 \%$ in this case, the magnitude reduction is no longer significant. This analysis proves that the damping ratio and hence the material chosen for the oscillators can influence the SOA's performance significantly. In cases where one has to select oscillators with low damping ratios for different design situations, SOAs with a higher number of oscillators can produce the desired frequency response [9].
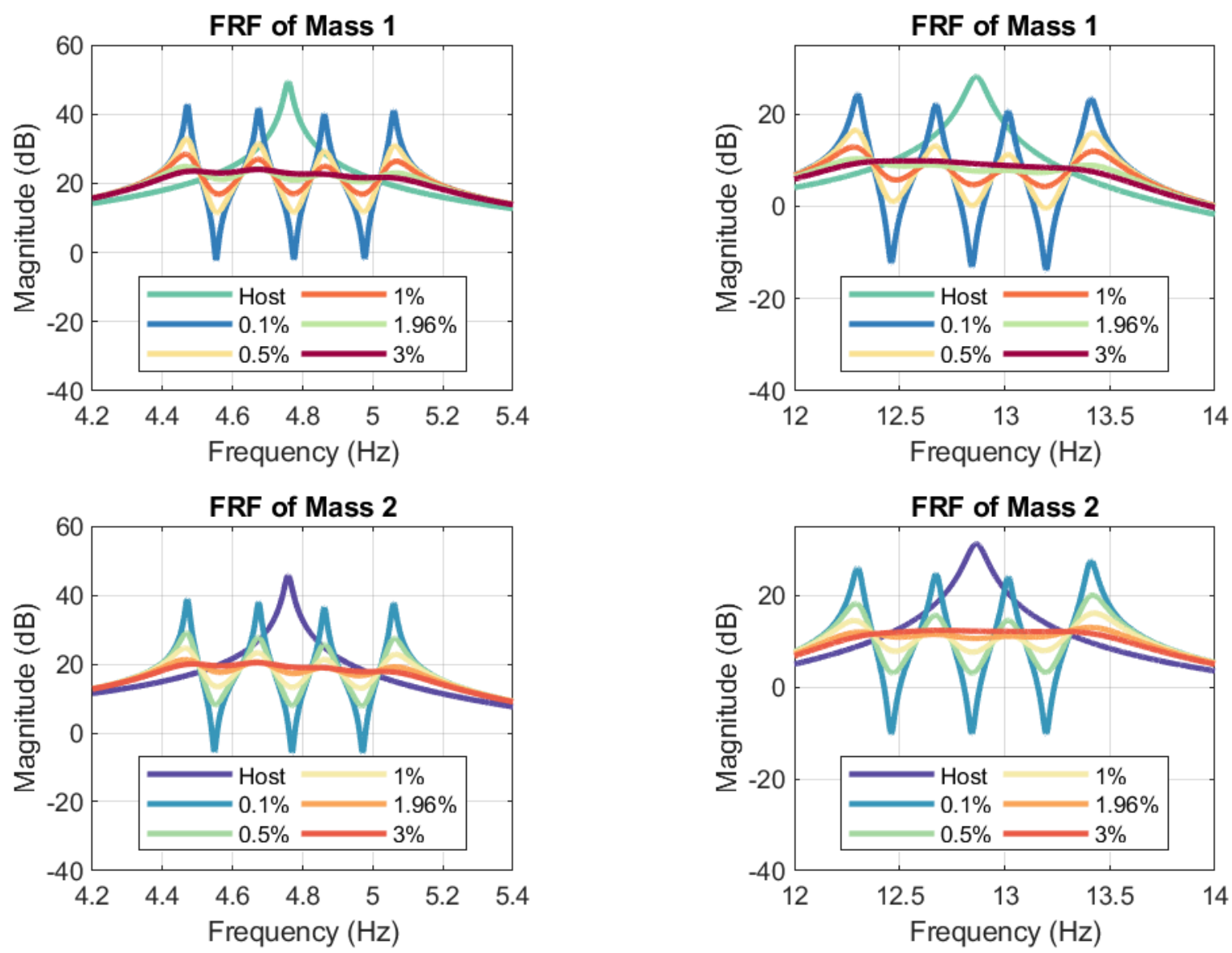

(a) First Resonant Frequency

(b) Second Resonant Frequency

Figure 10: Effect of damping on the FRFs of the host with SOAs

\subsection{Robustness Study}

Previous sections highlighted that SOAs are useful for broadband vibration attenuation, and it is known that single oscillator absorbers/DVAs are meant for cases where the frequency of excitation is relatively constant. In this section, the performance of SOAs and DVAs are investigated when the dynamic properties of the host are perturbed. The focus of this study is to investigate the performance of DVAs and SOAs when the natural frequency shifts from $\omega$ to $\tilde{\omega}$. Figures 11 and 12 compare the robustness of the SOA with that of the DVA. These figures present two ways of comparing the performance of SOAs and DVAs when the host's natural frequency is varied by $\pm 10 \%$, i.e., $0.9 \leq \tilde{\omega} / \omega \leq 1.1$. The $x$-axis of 
these figures is the perturbed non-dimensional natural frequency (i.e. $\tilde{\omega} / \omega$ ), and the value of 1 on the $\mathrm{x}$-axis corresponds to the unperturbed case discussed in the previous sections.

In Figure 11, the $d B$ reduction is calculated between the peak magnitude of the unmodified host and the highest peak's magnitude of the modified/perturbed host. For the first and the second resonant frequency, $\omega$ is equal to $4.76 \mathrm{~Hz}$ and $12.86 \mathrm{~Hz}$, respectively, and the $d B$ reduction in the figure gives a measure of the minimum attenuation achieved around the corresponding host's resonant peaks. One can observe that the $d B$ reduction in the case of SOA is higher than DVA at $\tilde{\omega} / \omega=1$ ( the unperturbed case ), which conforms with the previous discussions and intuition. As the host's properties are perturbed, the performance of both the systems deteriorate. However, even at high levels of perturbation, the SOA's performance is always better than that of the DVA for $0.9 \leq \tilde{\omega} / \omega \leq 1.1$. The shaded region shown in the figure gives a figurative intuition of the level of perturbation required for the system with SOA to deteriorate to the best performance achieved by the DVA.

As DVAs have the best performance at their tuned frequency - $\omega$, Figure 12 presents the $d B$ reduction at $\omega$. Given that the host's natural frequency is perturbed to $\tilde{\omega}$, the DVA is mistuned and its performance deteriorates drastically as seen in this figure. However, SOA's magnitude reduction remains fairly constant over a higher perturbation. Beyond a critical perturbation, both the systems deteriorate drastically. It is easy to infer from both the figures that SOAs are better for structures with higher uncertainty. On the other hand, DVAs are a better choice only when the uncertainty in structural parameters of the host is almost insignificant or when the system is excited at a single natural frequency, which are highly unlikely in practical applications.

\section{Conclusion}

The present work aims to design a hands-on experiment that extends the teaching of broadband vibration attenuation to a class focused on structural dynamics. An array of dynamic vibration absorbers, also called Subordinate Oscillator Arrays, are developed using fettuccine pasta and modeling clay to extend the concept and even provide simplified exposure to modern day meta-structures. The SOAs are designed to attenuate the dynamics of a 2-DOF structure. The dynamics of the modified structure is studied via simulations and experimental investigation.

\section{Acknowledgment}

Tarazaga would like to acknowledge the support provided by the John R. Jones III Faculty Fellowship.

\section{References}

[1] H. Frahm, Device for damping vibrations of bodies., uS Patent 989,958 (Apr. 18 1911).

[2] M. Strasberg, D. Feit, Vibration damping of large structures induced by attached small resonant structures, The Journal of the Acoustical Society of America 99 (1) (1996) 335-344. doi : 10. $1121 / 1.414545$.

URL https: //doi .org/10.1121/1.414545

[3] A. Akay, Z. Xu, A. Carcaterra, I. M. Koc, Experiments on Vibration Absorption using Energy Sinks, The Journal of the Acoustical Society of America 118 (5) (2005) 3043. doi : 10.1121/1.2046767.

[4] L. Zuo, S. A. Nayfeh, Optimization of the Individual Stiffness and Damping Parameters in Multiple-Tuned-Mass-Damper Systems, Journal of Vibration and Acoustics 127 (1) (2005) 77. 
FRF of Peak 1(MASS 1)

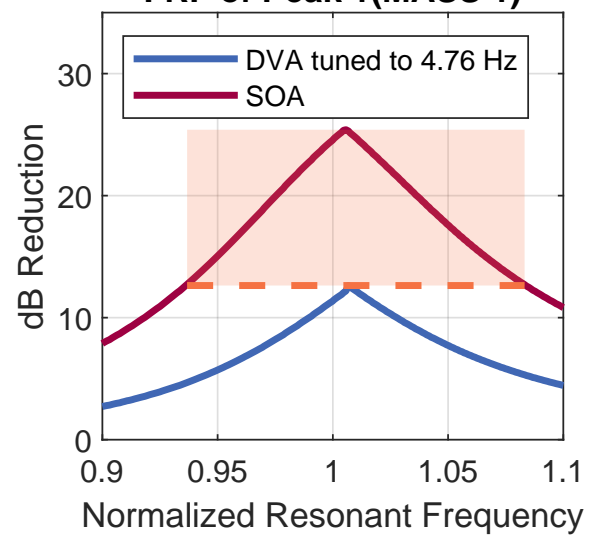

FRF of Peak 1 (MASS 2)

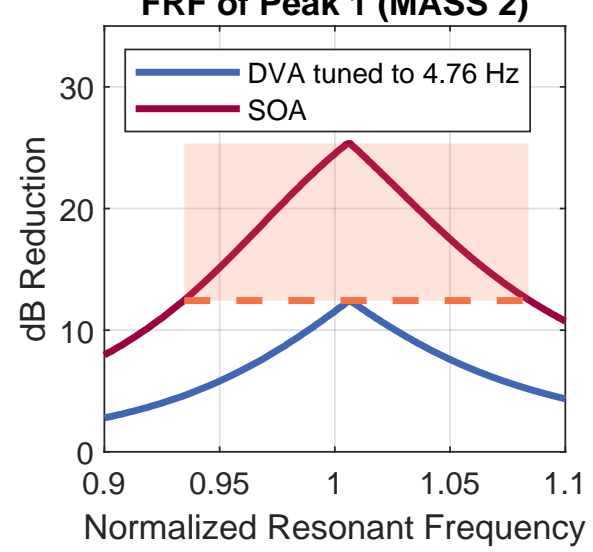

FRF of Peak 2(MASS 1)

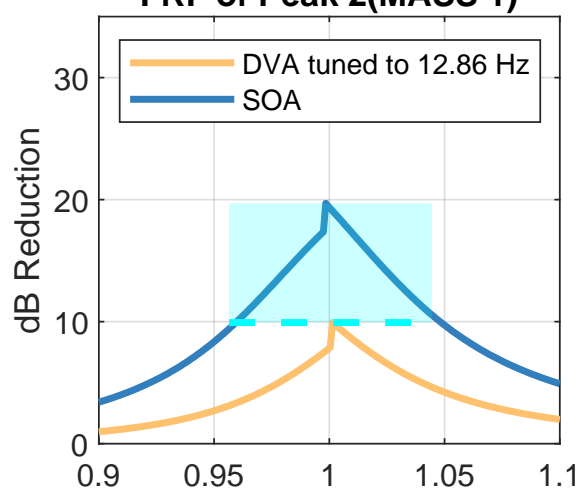

Normalized Resonant Frequency

FRF of Peak 2(MASS 2)

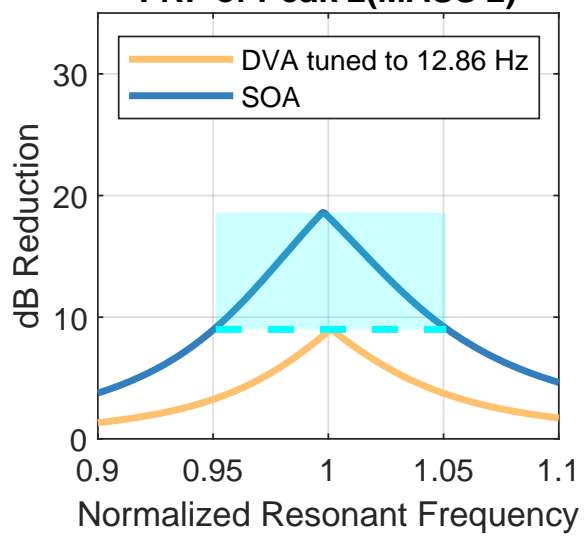

Figure 11: Variation of (minimum) $d B$ reduction between the peaks of unmodified and modified host (across a band) with $\tilde{\omega} / \omega$

doi: $10.1115 / 1.1855929$.

URL http://vibrationacoustics.asmedigitalcollection.asme.org/article.aspx? articleid $=1470725$

[5] A. Carcaterra, A. Akay, C. Bernardini, Trapping of vibration energy into a set of resonators: Theory and application to aerospace structures, Mechanical Systems and Signal Processing 26 (1) (2012) 1-14. doi: 10.1016/j.ymssp. 2011.05.005.

URL http://dx.doi.org/10.1016/j.ymssp.2011.05.005

[6] J. F. Vignola, J. A. Judge, A. J. Kurdila, Shaping of a system's frequency response using an array of subordinate oscillators, The Journal of the Acoustical Society of America 126 (1) (2009) 129-139. doi : $10.1121 / 1.3143783$.

URL http://asa.scitation.org.ezproxy.lib.vt.edu/doi/pdf/10.1121/1. 3143783https://doi.org/10.1121/1.3143783

[7] J. Vignola, J. Judge, J. Sterling, T. Ryan, A. Kurdila, S. T. Paruchuri, A. Glean, On the Use of Shunted Piezo Actuators for Mitigation of Distribution Errors in Resonator Arrays, in: Proceedings of the 22nd International Congress on Acoustics, 2016. 

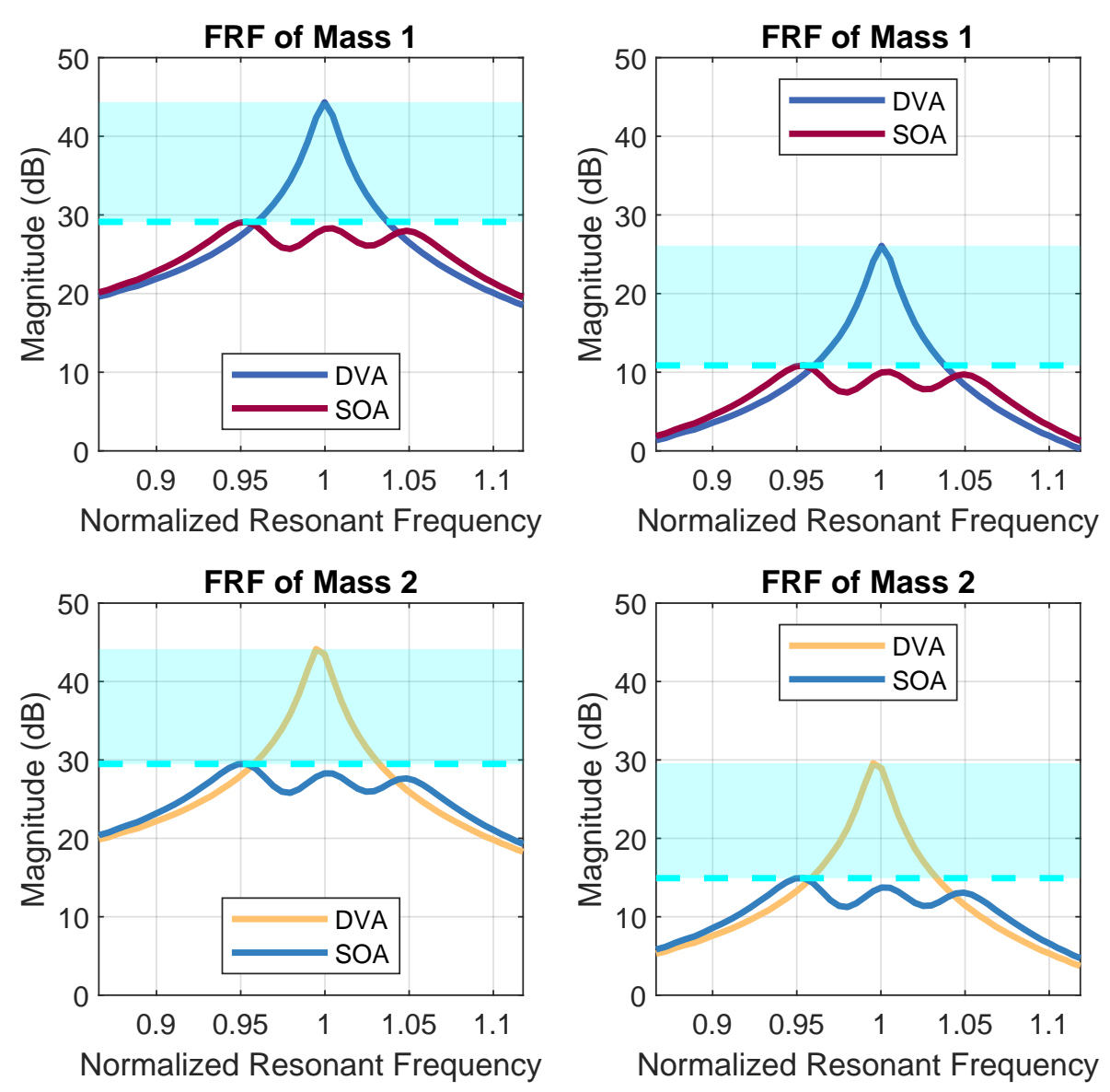

Figure 12: Variation of $d B$ reduction between the peaks of unmodified and modified host at host's natural frequency with $\tilde{\omega} / \omega$

[8] S. T. Paruchuri, A. J. Kurdila, J. Sterling, A. Vignola, J. Judge, J. Vignola, T. Ryan, Thermodynamic Variational Formulations of Subordinate Oscillator Arrays (SOA) With Linear Piezoelectrics, in: ASME. International Design Engineering Technical Conferences and Computers and Information in Engineering Conference, Volume 8: 29th Conference on Mechanical Vibration and Noise :V008T12A068., 2017. doi : 10.1115/DETC2017-68056.

URL http://dx. doi .org/10.1115/DETC2017-68056

[9] S. T. Paruchuri, J. Sterling, A. Kurdila, J. Vignola, Piezoelectric composite subordinate oscillator arrays and frequency response shaping for passive vibration attenuation, in: 2017 IEEE Conference on Control Technology and Applications (CCTA), IEEE, 2017, pp. 702-707. doi : 10 . 1109/CCTA . 2017.8062544.

URL http://ieeexplore.ieee.org/document/8062544/

[10] S. T. Paruchuri, J. Sterling, V. V. N. S. Malladi, A. Kurdila, J. Vignola, P. Tarazaga, Passive piezoelectric subordinate oscillator arrays, Smart Materials and Structures 28 (8) (2019) 85046. doi : 10.1088/1361-665x/ab2f5a.

URL https://iopscience.iop.org/article/10.1088/1361-665X/ab2f5ahttp: //dx.doi.org/10.1088/1361-665X/ab2f5a

[11] D. J. Inman, Engineering Vibration, Vibration Fundamentals and Practice (2001) 560arXiv: 
arXiv: 1011.1669v3, doi : 10.1016/B978-0-12-387000-1.01001-9.

URL http: //www . mendeley . com/research/engineering-vibration/

[12] R. R. Craig, A. J. Kurdila, Fundamentals of structural dynamics, John Wiley \& Sons, 2006.

[13] K. K. Reichl, D. J. Inman, Lumped mass model of a 1d metastructure for vibration suppression with no additional mass, Journal of Sound and Vibration 403 (2017) 75-89.

[14] S. Ford, T. Minshall, 3d printing in teaching and education: A review of where and how it is used, Additive Manufacturing.

[15] L. N. Virgin, Enhancing the teaching of elastic buckling using additive manufacturing, Engineering Structures 174 (2018) 338-345.

[16] L. Virgin, Enhancing the teaching of linear structural analysis using additive manufacturing, Engineering Structures 150 (2017) 135-142.

[17] L. Virgin, Enhancing the teaching of structural dynamics using additive manufacturing, Engineering Structures 152 (2017) 750-757.

[18] S. Calhoun, P. Harvey Jr, Enhancing the teaching of seismic isolation using additive manufacturing, Engineering Structures 167 (2018) 494-503. 